J. Biosci., Vol. 18, Number 3, September 1993, pp 345-354. C Printed in India.

\title{
Thermophilic fungi: an assessment of their potential for growth in soil
}

\author{
A K RAJASEKARAN and R MAHESHWARI* \\ Department of Biochemistry, Indian Institute of Science, Bangalore 560 012, India
}

MS received 5 March 1993; revised 25 May 1993

\begin{abstract}
An attempt has been made to forecast the potential of thermophilic fungi to grow in soil in the laboratory and in the field in the presence of a predominantly mesophilic fungal flora at usual temperature. The respiratory rate of thermophilic fungi was markedly responsive to changes in temperature, but that of mesophilic fungi was relatively independent of such changes. This suggested that in a thermally fluctuating environment, thermophilic fungi may be at a physiological disadvantage compared to mesophilic fungi. In mixed cultures in soil plates, thermophilic fungi outgrew mesophilic fungi under a fluctuating temperature regime only when the amplitude of the fluctuating temperatures was small and approached their temperature optima for growth. An antibody probe was used to detect the activity of native or an introduced strain of a thermophilic fungus, Thermomyces lanuginosus, under field conditions. The results suggest that although widespread, thermophilic fungi are ordinarily not an active component of soil microflora. Their presence in soil most likely may be the result of the aerial dissemination of propagules from composting plant material.
\end{abstract}

Keywords. Thermophilic fungi; compost; aerial dissemination; spores; Thermomyces lanuginosus; autecology.

\section{Introduction}

Among the eukaryotes, only thermophilic fungi have the exceptional ability to grow at high temperatures of $50^{\circ}-60^{\circ} \mathrm{C}$ (Cooney and Emerson 1964). Some thirty species of thermophilic fungi are currently known, most of which originally were isolated from composts (Tansey and Brock 1978). Their prevalence in composts is explained on the basis that prolonged elevated temperatures, humid and aerobic conditions, and supply of carbohydrate and nitrogen in the mass of organic matter favours the development of thermophilic microflora (Cooney and Emerson 1964). However, it is not certain if composts can be considered as 'natural' sites where thermophilic fungi may have evolved. Therefore natural habitats of thermophilic fungi have been searched, the soil being the principal among them. Although numerically far less than in composts, soils from virtually any place, when analysed by plating technique, have yielded thermophilic fungi (Apinis and Pugh 1979; Gochenaur 1975; Huang and Schmitt 1975; Maheshwari et al 1987). As the opacity of soil precludes in situ detection of fungal growth by light microscopy, indirect approaches were taken to verify whether their presence in soil was due to growth in situ or due to aerial dissemination of spores from foci of growth elsewhere and their fall-out from the atmosphere. Eggins et al (1972) used an immersion perfusion technique to screen fungi growing in soil and reported widespread occurrence of thermophilic fungi in soil, remaining dormant in shaded soil but becoming active in sun-heated

\footnotetext{
* Corresponding author.

Abbreviation used: FA, Fluorescent antibody.
} 
soil. Jack and Tansey (1977) studied development of thermophilic fungi in pure cultures in Petri dishes which had been sealed and then buried in field soil. All species tested germinated and sporulated under these conditions, leading the authors to the view that the extent and duration of elevated temperatures reached in sun-heated soil are sufficient for thermophilic fungi to complete life cycle in soil. However, inasmuch as thermophilic fungi occur in soil amidst a vast majority of mesophilic microflora and microfauna comprising potential competitors, antagonists and predators, the above opinion based on axenic cultures has remained doubtful.

To assess the growth of thermophilic fungi in soil at usual temperature, we have taken different experimental approaches. The influence of temperature on respiratory rates of mycelia of thermophilic and mesophilic fungi was compared to forecast their behaviour under fluctuating soil temperatures. The growth of a mixed population of mesophilic and thermophilic fungi was studied in soil plates at diurnally changing temperature in a programmed incubator to predict their competitive ability under natural conditions. An immunofluorescence technique was used to visualize the behaviour of spores of a thermophilic fungus in soil. The results are presented in this paper.

\section{Materials and methods}

\subsection{Organisms}

The following fungi, taken from our laboratory collection, were used in diferent experiments: Thermomyces lanuginosus, Penicillium duponti and Sporotrichum thermophile (thermophilic fungi); Aspergillus niger, Aspergillus phoenicis, Penicillium notatum and Trichoderma viride (mesophilic fungi).

\subsection{Measurement of respiration}

Thermophilic and mesophilic fungi were grown at $50^{\circ}$ and $30^{\circ} \mathrm{C}$, respectively. All media incorporated $2 \%$ glucose as the carbon source. Cultures were grown in $500 \mathrm{ml}$ Erlenmeyer flasks containing $150 \mathrm{ml}$ medium on a gyratory shaker $(240 \mathrm{rpm})$. The mycelia were harvested by filtration at the time when the growth reached half the maximal biomass. $T$. lanuginosus and $T$. viride were grown in a glucose-asparagine medium (Maheshwari and Balasubramanyam 1988) and used after 16 and $24 \mathrm{~h}$, respectively, $P$. duponti was grown for $24 \mathrm{~h}$ in the above medium supplemented with $0.01 \%(\mathrm{w} / \mathrm{v})$ yeast extract. A. phoenicis was grown for $18 \mathrm{~h}$ in a mineral salt solution. Washed mycelia were suspended in $50 \mathrm{mM}, \mathrm{pH} 5.6$, sodiumpotassium phosphate buffer and used for Warburg manometry as described previously (Prasad et al 1979). Respiration was measured in the presence of added glucose $(2 \%, \mathrm{w} / \mathrm{v})$.

\subsection{Soil plating}

Soils were collected from different places in India: Bangalore, Karnataka; Kota, Rajasthan; Pachmarhi, Madhya Pradesh; and Darjeeling, West Bengal. Approximately $500 \mathrm{mg}$ soil was sprinkled on solidified YpSs agar (Cooney and Emerson 
1964) which contained streptomycin sulphate $\left(50 \mathrm{mg}^{-1}\right)$ and rose bengal $(60 \mathrm{mg}$ $\left.1^{-1}\right)$. Each sample of soil was replicated six times. Incubation was for up to 1 week at constant temperature or at a fluctuating temperature regime in a programmable incubator (Model 124L Plant Growth Chamber, Controlled Environment Ltd., Winnipeg, Canada).

\subsection{Terminology}

Visibly distinct fungal colonies, isolated on soil plates, were categorised on the basis of their temperature requirements for growth on YpSs agar: (i) thermophilic, by its ability to grow at $48^{\circ}-50^{\circ} \mathrm{C}$ but not at $25^{\circ} \mathrm{C}$, (ii) thermotolerant, by its ability to grow at temperatures from $25^{\circ}$ to $50^{\circ} \mathrm{C}$, and (iii) mesophilic, by its growth at $25^{\circ} \mathrm{C}$ but not at $48^{\circ}-50^{\circ} \mathrm{C}$. The temperature tests were not done when the cultures could be identified correctly by morphological characters.

\subsection{Preparation of fluorescent-labelled antibody for immunofluorescence microscopy of T. lanuginosus}

Spores of $T$. lanuginosus strain RM-B were incubated in a glucose-asparagine medium at $50^{\circ} \mathrm{C}$ for $9 \mathrm{~h}$ on a shaker. Washed germlings were suspended in sterile $0 \cdot 85 \% \mathrm{NaCl}$ at $2 \mathrm{mg}$ wet wt $\mathrm{ml}^{-1}$ and used as the antigen. Male rabbits were injected intravenously with $1 \mathrm{ml}$ of the fungal suspension at intervals of 1 week for 4 weeks. The rabbits were rested for two weeks after the final injection before blood samples were collected from the ear vein. Antibody production was determined by agglutination test with spores. The maximum agglutination titer was 1/2048.

A fluorescent antibody probe was prepared by coupling fluorescein isothiocyanate to goat anti-rabbit immunoglobulin (IgG). For indirect immunofluorescence microscopy, $100 \mu \mathrm{l}$ of 1:50 diluted antibody was placed over the sample on cover slips. After incubation at room temperature for $1 \mathrm{~h}$, the sample was rinsed in 5 changes of $0.1 \mathrm{M}, \mathrm{pH} 6 \cdot 8$, sodium-potassium phosphate buffer containing $0.9 \%$ $\mathrm{NaCl}$. The sample was treated with 1:50 diluted fluorescein-labelled anti-rabbit IgG. After incubation for $30 \mathrm{~min}$, the sample on the cover slip was again rinsed in the buffer-saline solution, mounted in $70 \%$ glycerol and viewed under UV light using a Leitz Ortholux microscope.

\subsection{Detection of T. lanuginosus in soil}

The growth of $T$. lanuginosus in soil was studied by a modification of the buriedSlide technique (Schmidt and Bankole 1963). Clean cover slips (20 by $20 \mathrm{~mm}$ ) and those with $T$. lanuginosus spores adhering to them were buried in soil at different sites in a field. The spores were adhered to the cover slips by gently warming a smear of an aqueous suspension after checking that this procedure did not affect their viability. At least 20 cover slips were buried per site at a depth of approximately $3 \mathrm{~cm}$ in loosened soil in shaded and exposed areas. Replicate cover slips were also placed in soil in Petri dishes in the laboratory to monitor germination. At intervals, the cover slips were removed, rinsed gently in sterile water and examined microscopically using phase-contrast or UV optics. 


\subsection{Soil temperature}

Soil temperature in the field was measured using a thermistor probe (Yellow Springs Co., Yellow Springs, Ohio, USA) connected to a battery-powered stripchart recorder.

\subsection{Sampling of air-borne spores}

A volumetric, rotating-arm impaction device (Model 82 Rotorod Sampler, Sampling Technologies Inc., Los Altos, California, USA) was used to sample airborne spores, 2 feet above the ground. The collector rods were coated with a jelly mixture containing $4 \%$ gelatin, $50 \%$ glycerine and $0 \cdot 1 \%$ Tween 20 . After use, the rods were placed in sterile water to remove spore deposit and aliquots of aqueous suspension were plated on $\mathrm{YpSs}$ agar at $50^{\circ} \mathrm{C}$ for isolation and identification of thermophilic fungi.

\section{Results}

\subsection{Effect of temperature on respiratory activity of fungi}

To determine whether mesophilic and thermophilic species can maintain their metabolic rate during temperature fluctuations of the environment, the respiratory rates of fungal mycelia were measured over a range of temperature. These data are given as Arrhenius plot of the logarithm of respiratory rate $\left(Q_{\mathrm{o}_{2}}\right)$ against the reciprocal of the absolute temperature (figure 1). The maximal $Q_{\mathrm{O}_{2}}$ of thermophilic fungi (T. lanuginosus and $P$. duponti) and of mesophilic fungi (T. viride and $A$. phoenicis) occurred, respectively, at $50^{\circ} \mathrm{C}$ and $30^{\circ}-40^{\circ} \mathrm{C}$. The $Q_{\mathrm{o} 2}$ of mesophilic fungi was higher than that of thermophilic fungi over a wide range of temperatures.

\subsection{Competitive growth of mesophilic and thermophilic fungi at fluctuating temperature regimes}

The type of fungus - mesophilic or thermophilic — that would grow from propagules in soil when it is plated on YpSs agar and incubated under a fluctuating temperature regime was determined. It was first ascertained that the soils tested contained both types of fungi. The control soil plates were incubated at constant $28^{\circ} \mathrm{C}$ or at constant $48^{\circ} \mathrm{C}$. Incubation at $28^{\circ} \mathrm{C}$ yielded colonies of mesophilic fungi of which the following were dominant species: Aspergillus spp. (mostly black aspergilli), Mucor sp. and Penicillium spp. Incubation at $48^{\circ} \mathrm{C}$ yielded one or more species of the following thermophilic fungi in every soil tested: Chaetomium thermophile La Touche, Humicola grisea Traaen, Malbranchea pulchella Saccardo and Penzing var. sulfurea (Miehe) Cooney and Emerson, Sporotrichum thermophile Apinis, Thermoascus aurantiacus Miehe, Thermomyces lanuginosus Tsiklinsky and Torula thermophila Cooney and Emerson. Among the thermophilic fungi, $T$. lanuginosus was the most common in soil plates. Quantitation of fungal colonies on the soil plates incubated at $48^{\circ}$ or $28^{\circ} \mathrm{C}$ was not done, but there was an obvious 


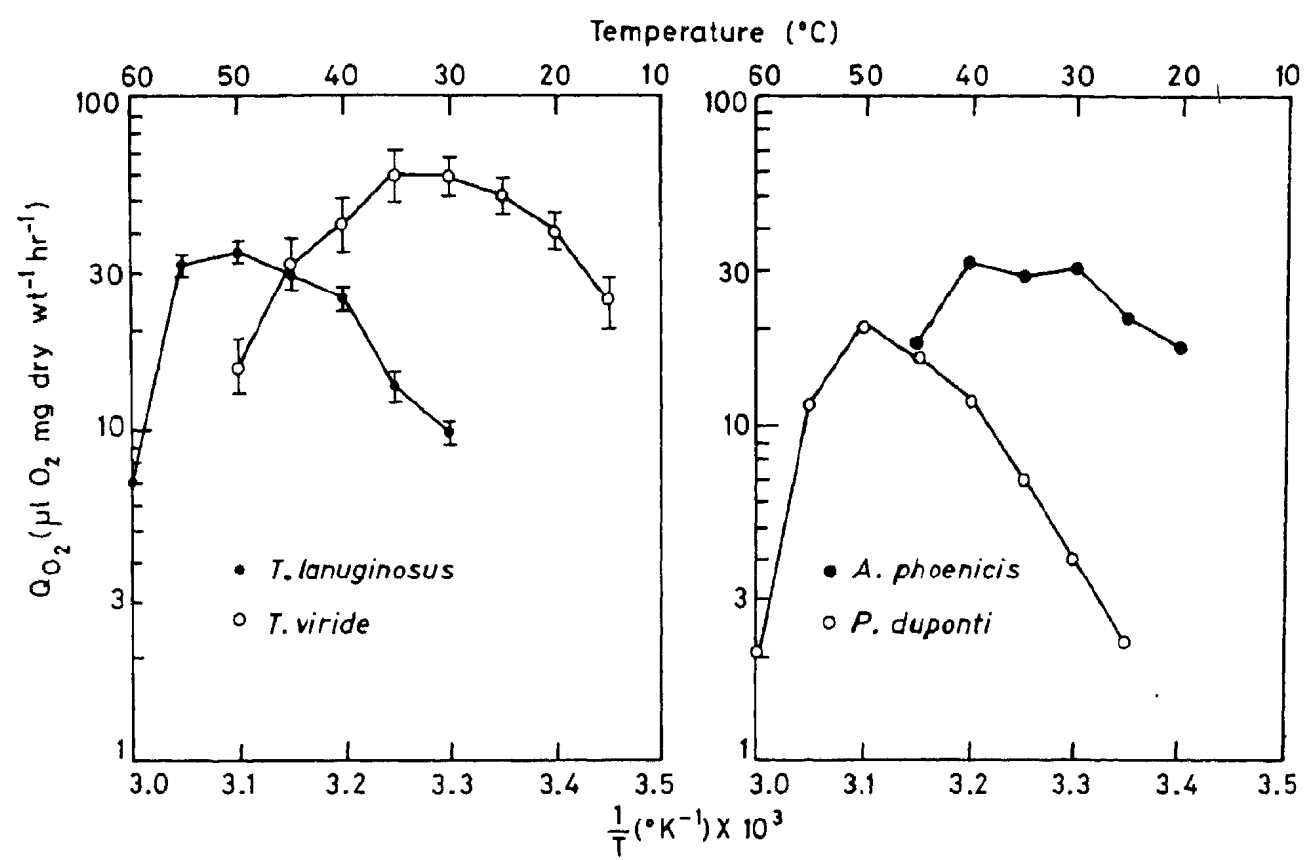

Figure 1. Arrhenius plots of respiratory rates of mesophilic and thermophilic fungi at different temperatures. The data for T. lanuginosus (thermophilic) and T. viride (mesophilic) are average values $\pm \mathrm{SE}$ from three experiments. The data for $P$. duponti and A. phoenicis are average values of triplicate Warburg flasks from a single experiment.

excess of mesophilic fungi over thermophilic fungi showing that soil contained higher number of mesophilic propagules.

Incubation at fluctuating temperature regimes, from $24^{\circ}$ to $48^{\circ} \mathrm{C}, 32^{\circ}$ to $48^{\circ} \mathrm{C}$ and from $36^{\circ}$ to $48^{\circ} \mathrm{C}$ (figure 2) yielded mesophilic fungi or a thermotolerant fungus identifiable as Aspergillus fumigatus. At the fluctuating temperature regime, from $36^{\circ}$ to $48^{\circ} \mathrm{C}$, the thermophilic fungus $H$. grisea var. thermoidea was also present in some soil plates. By contrast, at the fluctuating temperature regime of $40^{\circ}$ to $48^{\circ} \mathrm{C}$, only thermophilic fungi appeared in soil plates; $T$. lanuginosus being the most common.

It was determined whether increasing the number of propagules of a thermophilic fungus in soil can improve its recovery in soil plates at the temperature regimes which were unproductive in the above experiment. Approximately $10^{8}$ spores of $T$. lanuginosus were thoroughly mixed with $50 \mathrm{~g}$ of garden soil and a portion of it was plated. Approximately 2-4 times more colonies of $T$. lanuginosus were recovered from the enriched soil than from the control, but again when incubation temperature varied between $40^{\circ} \mathrm{C}$ (minimum) and $48^{\circ} \mathrm{C}$ (maximum) only. The results showed that temperature of environment was an important factor determining growth of thermophilic fungi in soil plates.

Growth of $T$. lanuginosus was also tested in pure culture at the temperature regimes which were unfavourable in soil-plate experiment. Spores seeded on YpSs agar and incubated at any of the fluctuating temperature regimes developed colonies. This showed that the temperature of the fluctuating cycle per se can support germination and development of T. lanuginosus provided it is present alone. 


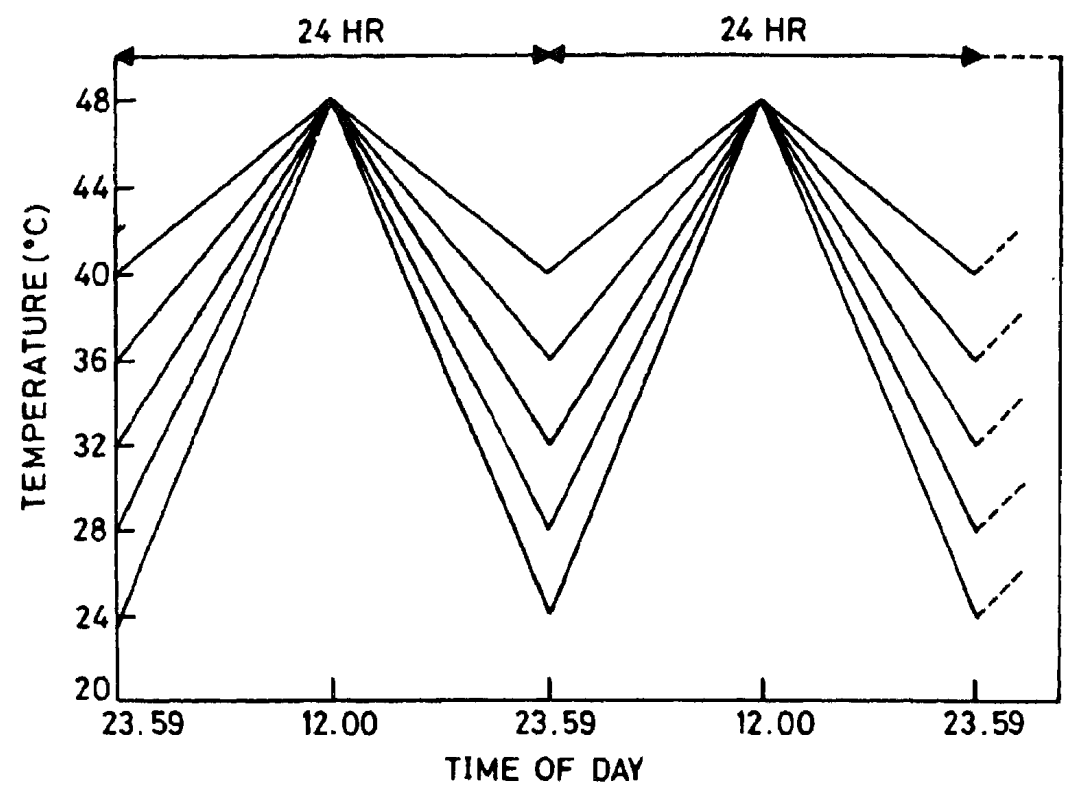

Figure 2. Diagram of five diurnally fluctuating temperature regimes used to programme controlled environment chamber for study of the competitive growth of a mixed soil fungal flora on nutrient media.

\subsection{Autecology of T. lanuginosus by immunofluorescence microscopy}

Immunofluorescence was used to study the autecology of $T$. lanuginosus in soil This fungus was chosen because it is the most ubiquitous thermophilic fungus in soil (Maheshwari et al 1987). First, the specificity and the efficacy of the fluorescent antibody (FA) preparation was tested. T. lanuginosus mycelium treated with preimmunized serum did not fluoresce. The FA reacted strongly with strain RM-B and five other strains of the same fungus, showing that the antibody was not strainspecific. The FA reacted also with mycelia of $A$. niger grown in axenic culture but not with those of the four other fungi tested: P. duponti, S. thermophile, P. notatum and $T$. viride. The results showed that FA was sufficiently, but not absolutely, specific to T. lanuginosus.

In order to learn whether the FA preparation would detect $T$. lanuginosus if present in soil, spores were adhered to glass cover slips and incubated under nonsterile garden soil in Petri dishes kept in incubators at $30^{\circ}, 50^{\circ} \mathrm{C}$ or at a temperature regime which fluctuated between $40^{\circ}$ and $48^{\circ} \mathrm{C}$. At $30^{\circ} \mathrm{C}$, spores remained dormant up to three weeks when the observations were terminated. In contrast, at $50^{\circ} \mathrm{C}$ spores germinated in $4 \mathrm{~h}$ (figure 3A). The hyphal growth on cover slips reacted positively with the FA preparation and produced a bright apple-green fluorescence (figure 3B). The wall of the germinated spore did not react in immunofluorescence although the antiserum agglutinated the ungerminated spores. Presumably, the antigen from the spore wall was lost during germination. Spores germinated also at the fluctuating temperature regime (figure $3 \mathrm{C}$ ) and the germ tubes gave a positive reaction with FA (not shown). The results showed that $T$. lanuginosus can grow in soil and maintain its antigenicity. 

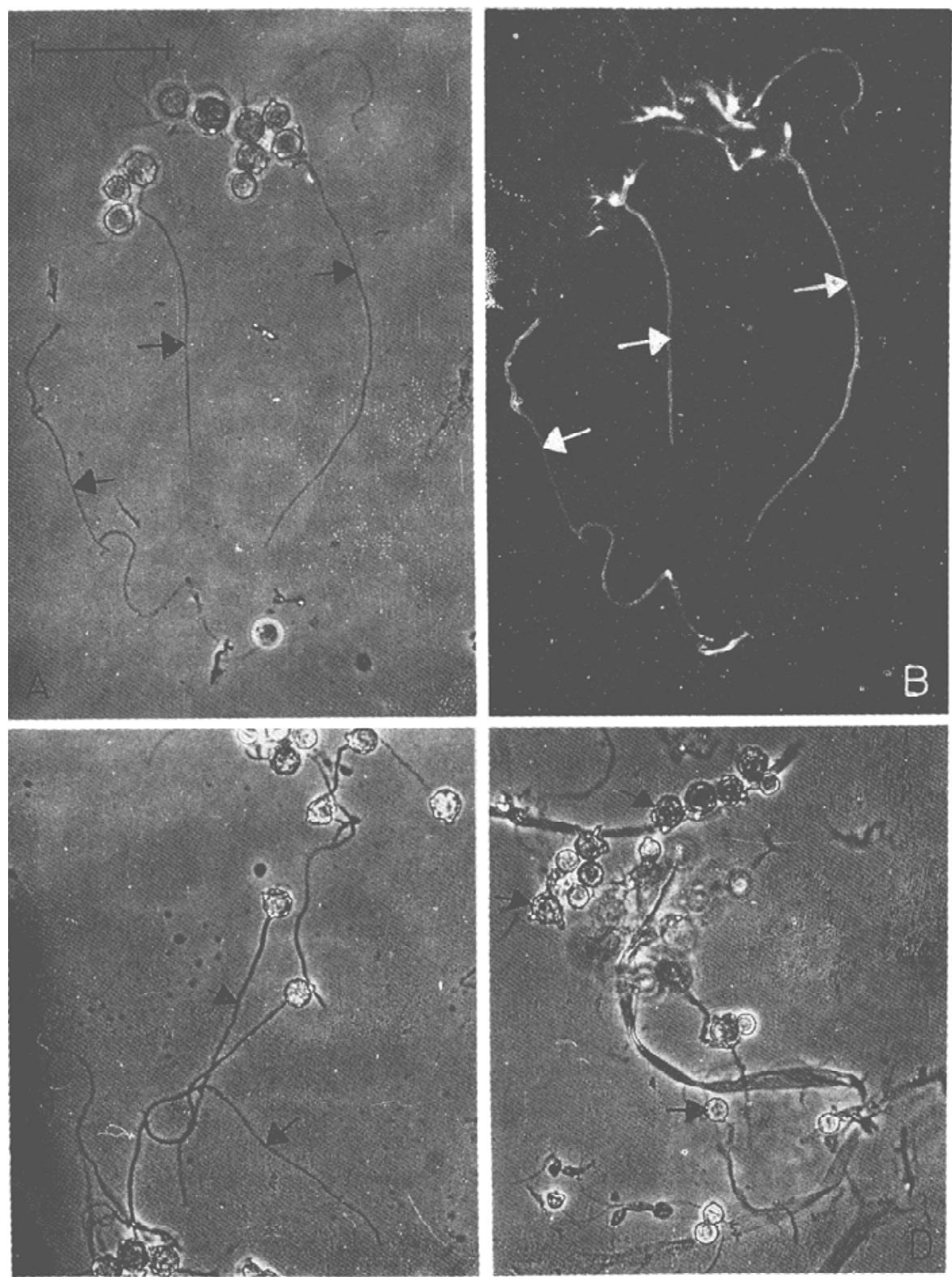

Figure 3. Microscopic examination of T. lanuginosus spores on cover-slips. (A) Germinating spores $(\uparrow)$ on cover-slip placed in contact with soil in Petri dish in a constant $50^{\circ} \mathrm{C}$ incubator for $4 \mathrm{~h}$ and stained with fluorescent-antibody. Phase contrast microscopy. (B) Same as viewed under UV showing fluorescence of hyphal growth ( $\uparrow$ ) (C) Cover-slip bearing spores of $T$. lanuginosus in contact with soil in Petri dish in an incubator operating on a diurnally fluctuating thermal regime $\left(\min .40^{\circ} \mathrm{C}\right.$, max. $48^{\circ} \mathrm{C}$ ) for $4 \mathrm{~h}$. Germination of spores $(\uparrow)$ has occurred. Phase contrast microscopy. (D) Phase contrast microscopy of cover-slip bearing $T$. lanuginosus spores $(\uparrow)$ buried in soil in the field for 8 days and treated with a fluorescent-antibody. Dormant spores of $T$. lanuginosus and unidentified growth are seen. The hyphal growth did not show fluorescence when viewed under UV light. All micrographs are approximately of same magnification. Bar length is $50 \mu \mathrm{m}$. 
Based on the above results, an attempt was made to detect $T$. lanuginosus in a field. Samples of soil from the experimental sites were plated to confirm the presence of $T$. lanuginosus. Phase-contrast microscopy showed mycelial growth on cover slips which had been buried in field for over three weeks. On some cover slips sporulating mycelia of Alternaria sp. and of an actinomycete were present which did not react with FA. Mycelia which reacted positively with FA were not found.

The concentration of the resident population of T. lanuginosus in soil could be too low for successful competition and detection. Therefore, spores of T. lanuginosus strain RM-B were adhered on cover slips and introduced into shaded and exposed areas in the field. These spores remained dormant for up to three weeks when observations were terminated. However, some hyphal growth was observed but it was not produced from spores of $T$. lanuginosus adhering to the cover slips (figure 3B). This fungal growth reacted negatively with FA in most samples. In a few cover slips, however, the hyphal fragments stained with FA but since reproductive structures were absent, these could not be identified as belonging to T. lanuginosus. The diameter of the hyphal fragments appeared too wide and the cell wall exceptionally thick and as a result these hyphae were not considered to be $T$. lanuginosus. The above experiments with the buried cover slips were done during June-September when the moisture levels in the soil seemed favourable for microbial activity as evidenced by decomposing substrates in the field. The soil temperature during the experiments varied between $22^{\circ}-28^{\circ} \mathrm{C}$.

\subsection{Detection of spores of thermophilic fungi in atmosphere}

Sampling of air in the field by Rotorod Sampler yielded thermophilic fungi. $T$. lanuginosus and three unidentified fungi were recovered, showing the presence of thermophilic fungi in air.

\section{Discussion}

The Arrhenius plots of $Q_{\mathrm{O}_{2}}$ of mycelia as a function of temperature indicate that the metabolic rate of mesophilic fungi is affected less by the changes in temperature than that of thermophilic fungi (Prasad et al 1979; present study). The biochemical basis of this difference in the thermal responses of mycelia of mesophilic and thermophilic fungi has not been investigated; nevertheless this behaviour may have implications for growth in natural conditions. If mycelia in soil environment respond similarly (figure 1), then the fungi which can maintain optimal metabolic rate over a broad range of temperatures may be expected to have a competitive advantage over those which lack this ability. Mesophilic fungi, rather than thermophilic fungi, would appear to be better adjusted to soil where temperatures vary both spatially and temporally.

This inference is corroborated by the results of soil plating at the fluctuating temperatures. Mesophilic fungi developed in soil isolation plates when the minimum temperature was varied from $24^{\circ}-36^{\circ} \mathrm{C}$ to a maximum of $48^{\circ} \mathrm{C}$. Thermophilic fungi appeared in soil plates only when the incubation temperature fluctuated to a small extent (between $40^{\circ}$ and $48^{\circ} \mathrm{C}$ ) approaching the average optimum growth temperature of $46^{\circ} \mathrm{C}$ of thermophilic fungi (Cooney and Emerson 1964). Although soil in some places does heat up to $40^{\circ}-50^{\circ} \mathrm{C}$ or more (data 
provided by Metereological Survey of India, Pune; Stotzky 1974), the important point is whether adequate moisture is available when soil temperatures conducive for thermophile growth are attained. Cooney and Emerson (1964) emphasized moisture as one of the factors of crucial importance in development of thermophilic microflora.

The thermophile $T$. lanuginosus is one of the most common fungi occurring in soil (Maheshwari et al 1987). However, immunofluorescence microscopy did not detect the growth of either the indigenous or of the introduced strain of this fungus on cover slips buried in the field. Because of the problems encountered in observing and identifying propagules in soil itself, we used glass cover slips with or without spores of T. lanuginosus adhered to it as the support. It may, however, be that mycelia of T. lanuginosus grow in between crevices of soil particles and glass cover slips did not provide a suitable surface which acted as a barrier for diffusion of water and nutrients for initiation of growth. This problem was minimized by using the buried cover slips during the monsoon season with moisture levels in soil favourable for microbial activity in the field. Since $T$. lanuginosus spores placed on cover slips in soil did germinate at $50^{\circ} \mathrm{C}$ in an incubator, it seems reasonable that low temperature rather than wrong surface, nutrient limitation or contact with inhibitory substances was the chief reason for the failure of spores to germinate in field conditions.

The results of all three experimental approaches used in this study suggest that thermophilic fungi are not a normally active component of microflora in the soil. In as far as is known thermophilic fungi are poorly represented also in thermal soils from volcanic regions (Cooney and Emerson 1964). How then may we explain the world-wide distribution of $T$. lanuginosus and other species of thermophilic fungi in soils (Ellis and Keane 1981; Maheshwari et al 1987)? It is significant that species of thermophilic fungi obtained from sampling of air, $6 \mathrm{~km}$ from a waste compost system in Bombay (Thakur 1977), were the same as those from over 200 soil samples from several places in India (Apinis and Pugh 1979; Maheshwari et al 1987). The presence of air-borne propagules of thermophilic fungi in temperate (Evans 1972; Hudson 1974; Hughes and Crossier 1973), subtropical or tropical countries (Hedger 1975; Sandhu and Singh 1985; Thakur 1977; present study) suggests that their wide distribution in soil is a result of the dissemination of their propagules from composts.

It may be asked if 'natural' composts existed before man where thermophilic fungi might have evolved? Cooney and Emerson (1964) suggested that nests of an old group of birds, the Megapodidae, or the incubator birds, which have probably existed since 50 to 60 million years (Grzimek 1984), as possible natural sites of occurrence of the thermophilic fungi. These birds occur in Australia and islands of the south western Pacific, and incubate their eggs inside large mounds (composts) which they construct out of forest litter wherein the microbial activity produces respiratory heat that warms them up to $33^{\circ}-50^{\circ} \mathrm{C}$ for several months (Seymour and Bradford 1992). Probably thermophilic fungi evolved from their mesophilic counterparts in such thermal, humid and aerobic environment.

\section{Acknowledgements}

We thank Professor (Emeritus) Alfred S Sussman, University of Michigan, for his 
valuable comments on this manuscript and suggestions on future work. This work was supported by the Department of Science and Technology, New Delhi.

\section{References}

Apinis A E and Pugh G J F 1979 Thermophilous fungi in Southern India; Phytocoenologia 6 152-165

Cooney D G and Emerson R 1964 Thermophilic fungi. An account of their biology, activities and classification (San Fransisco: W H Freeman)

Eggins H O W, von Szilvinyi A and Allsopp D 1972 The isolation of actively growing thermophilic fungi from insolated soils; Int. Biodeterior. Bull. 8 53-59

Ellis D H and Keane P J 1981 Thermophilic fungi isolated from some Australian soils; Aust. J. Bot. 29 689-704

Evans H C 1972 Thermophilous fungi isolated from air; Trans. Br. Mycol. Soc. 59 516-519

Grzimek B 1984 Animal life encyclopedia. Vol. 7, Birds I (New York: Van Nostrand Reinhold) pp 432443

Gochenaur S E 1975 Distribution of mesophilous and thermophilous microfungi in two Bahamian soils; Mycopathologia 57 155-164

Hedger J N 1975 The ecology of thermophilic fungi in Indonesia; in Biodegradation et humifcation (eds) G Kilbertus, O Reisinger, A Mourey and J A Cancela de Fonseca (Nancy; Pierron Sarreguemines) pp 59-65

Huang L H and Schmitt J A 1975 Soil microfungi of central and southern Ohio; Mycotaxon 3 55-80

Hudson H J 1974 Thermophilous and thermotolerant fungi in the air-spora at Cambridge; Trans. Br. Mycol Soc. 60 596-598

Hughes W T and Crossier J W 1973 Thermophilic fungi in the mycoflora of man and environmental air; Mycopathol. Mycol Appl. 49 147-152

Jack M A and Tansey M R 1977 Growth, sporulation and germination of spores of thermophilic fungi incubated in sun-heated soil; Mycologia 69 109-117

Maheshwari R, Kamalam P T and Balasubramanyam P V 1987 The biogeography of thermophilic fungi; Curr. Sci. 56 151-155

Maheshwari R and Balasubramanyam P V 1988 Simultaneous utilization of glucose and sucrose by thermophilic fungi; J. Bacteriol. $1703274-3280$

Prasad A R S, Kurup C K R and Maheshwari R 1979 Efect of temperature on respiration of a mesophilic and a thermophilic fungus; Plant Physiol 64 347-348

Sandhu D K and Singh S 1985 Air-borne thermophilous fungi at Amritsar, India; Trans. Br. Mycol Soc. 84 41-46

Schmidt E L and Bankole R O 1963 The use of fluorescent antibody with the buried slide technique; in Soil organisms (eds) J Doeksen and J van de Drift (Amsterdam: North Holland) pp 197-204

Seymour R S and Bradford D F 1992 Temperature regulation in the incubation mounds of the Australian brush turkey; The Condor 94 134-150

Stotzky G 1974 Activity, ecology and population dynamics of microorganisms in soil; in Microbial ecology (eds) A I Laskin and H Lechevalier (Cleveland: CRC Press) pp 57-135

Tansey M R and Brock T D 1978 Microbial life at high temperatures: Ecological aspects; in Life in extreme environments (ed.) D J Kushner (London: Academic Press) Chapter 5

Thakur S B 1977 Occurrence of spores of thermophilic fungi in the air at Bombay; Mycologia 69 197199 